\title{
Factors Influencing Sustained Engagement with ECG Self-Monitoring: Perspectives from Patients and Health Care Providers
}

\author{
Meghan Reading ${ }^{1}$ Dawon Baik ${ }^{2}$ Melissa Beauchem
${ }^{1}$ Division of Health Informatics, Department of Healthcare Policy and
Research, Weill Cornell Medicine, Cornell University, New York, \\ New York, United States \\ 2 School of Nursing, Columbia University, New York, New York, \\ United States \\ ${ }^{3}$ Department of Biomedical Informatics, Columbia University, \\ New York, New York, United States
}

Appl Clin Inform 2018;9:772-781.

Address for correspondence Meghan Reading, PhD, MPH, RN, Division of Health Informatics, Department of Healthcare Policy and Research, Weill Cornell Medicine, 425 East 61st Street, Suite 301, New York, NY 10065, United States (e-mail: mjr2011@med.cornell.edu).

\section{Abstract}

Keywords

- patient engagement

- patient-provider communication

- workflows and human interactions

- smart phone

- mobile platforms

- general information systems and technologies in clinics

- consumer health informatics
Background Patient-generated health data (PGHD) collected digitally with mobile health (mHealth) technology has garnered recent excitement for its potential to improve precision management of chronic conditions such as atrial fibrillation (AF), a common cardiac arrhythmia. However, sustained engagement is a major barrier to collection of PGHD. Little is known about barriers to sustained engagement or strategies to intervene upon engagement through application design.

Objective This article investigates individual patient differences in sustained engagement among individuals with a history of AF who are self-monitoring using mHealth technology. Methods This qualitative study involved patients, health care providers, and research coordinators previously involved in a randomized, controlled trial involving electrocardiogram (ECG) self-monitoring of AF. Patients were adults with a history of AF randomized to the intervention arm of this trial who self-monitored using ECG mHealth technology for 6 months. Semistructured interviews and focus groups were conducted separately with health care providers and research coordinators, engaged patients, and unengaged patients. A validated model of sustained engagement, an adapted unified theory of acceptance and use of technology (UTAUT), guided data collection, and analysis through directed content analysis.

Results We interviewed 13 patients (7 engaged, 6 unengaged), 6 providers, and 2 research coordinators. In addition to finding differences between engaged and unengaged patients within each predictor in the adapted UTAUT model (perceived ease of use, perceived usefulness, facilitating conditions), four additional factors were identified as being related to sustained engagement in this population. These are: (1) internal motivation to manage health, (2) relationship with health care provider, (3) supportive environments, and (4) feedback and guidance.

Conclusion Although it required some modification, the adapted UTAUT model was useful in understanding of the parameters of sustained engagement. The findings of this study provide initial requirement specifications for the design of applications that engage patients in this unique population of adults with AF. received

May 9, 2018

accepted after revision

August 19, 2018 (c) 2018 Georg Thieme Verlag KG Stuttgart · New York
DOI https://doi.org/

10.1055/s-0038-1672138. ISSN 1869-0327. 


\section{Background and Significance}

An increasing number of patients are using mobile health (mHealth) technology, including smartphones and other connected devices, to generate data that provide a rich account of their day-to-day health. ${ }^{1-3}$ These data, termed patient-generated health data (PGHD), may include physiologic measures, symptoms, and lifestyle data. ${ }^{4,5}$ PGHD has garnered excitement for its ability to uncover fluctuations in health-related factors that may play an important role in an individual's health and wellness. ${ }^{6-9}$ PGHD also is valuable for centering care on the patient and their unique environmental, lifestyle, and biological circumstances. ${ }^{6,10}$ As such, PGHD holds particular promise for precision management of individuals living with chronic conditions. ${ }^{11,12}$

One condition for which PGHD could be particularly valuable is atrial fibrillation (AF), the most common cardiac arrhythmia encountered in clinical practice. ${ }^{13} \mathrm{AF}$ is difficult to capture outside the clinical setting because it requires documentation via electrocardiogram (ECG) and is episodic and poorly correlated with patient-reported symptoms. ${ }^{14-16}$ Moreover, AF is deeply influenced by modifiable lifestyle factors such as alcohol use and obesity. ${ }^{17,18}$ Thus, PGHD can improve patient selfmanagement of the arrhythmia, while also offering clinical benefits to providers seeking to improve detection and tailor care based on the unique characteristics of the patient. ${ }^{19,20}$

Sustained patient engagement with self-monitoring using mHealth technology is necessary to generate adequate health data to enable precision management. ${ }^{21}$ Yet, evidence shows that patient engagement is low over time, with many abandoning self-monitoring within 3 to 6 months of initiation. ${ }^{22,23}$ There is a gap in understanding factors that contribute to sustained engagement, as much of the extant literature focuses solely on initial uptake of technology. ${ }^{24-26}$ Moreover, engagement research has had minimal success improving sustained engagement with generic design tactics, such as gamification and incentives (e.g., points, money), that forgo consideration of unique patient characteristics. ${ }^{27,28}$

\section{Objective}

The purpose of this study was to investigate individual differences in sustained engagement among patients with a history of AF who are self-monitoring using mHealth technology. Specifically, we aimed to uncover factors associated with sustained engagement through qualitative focus groups and interviews guided by a theoretical model.

\section{Methods}

\section{Theoretical Model}

Our investigation was guided by the unified theory of acceptance and use of technology (UTAUT) model. UTAUT is a comprehensive theory derived from eight models of behavior change and technology acceptance. Validation of the model found that it explains variation in technology acceptance and use better than its component models $\left(R^{2}=0.69\right.$ compared with $0.17-0.53){ }^{29}$ It has since been used in multiple health care studies. ${ }^{30-32}$ We chose UTAUT because it has been adapted specifically for sustained engagement. ${ }^{33}$ This adapted model guided our analysis ( - Fig. 1). In the adapted model, the predictors of sustained engagement with ECG mHealth technology are perceived ease of use, perceived usefulness, and three facilitating conditions tailored for our patient population: (1) AF knowledge, (2) AF symptoms severity, and (3) frequency of AF episodes. Age and gender moderate the relationships between all predictors and the outcome sustained engagement. Experience with technology moderates only the relationships of perceived ease of use and perceived usefulness with the outcome.

\section{Study Design and Sample}

This qualitative descriptive study ${ }^{34}$ used focus groups and individual interviews with patients, nurse practitioners and physicians (providers), and research coordinators involved in the iPhone Helping Evaluate Atrial Fibrillation Rhythm through Technology trial (iHEART; R01NR014853, PI: Hickey). This is an ongoing, 5-year randomized, controlled trial of adults with a

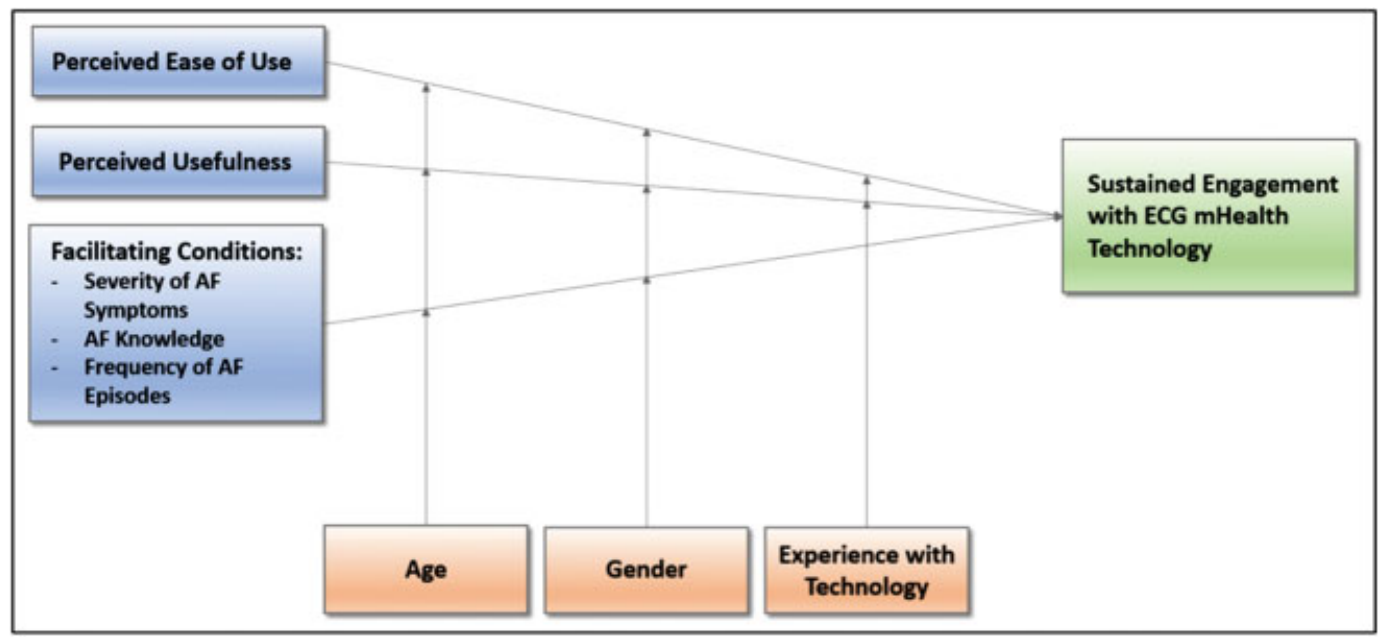

Fig. 1 Adapted unified theory of acceptance and use of technology model. AF, atrial fibrillation; ECG, electrocardiogram. 
history of AF who have undergone a procedure to restore a normal rhythm to the heart. ${ }^{35}$ They are randomized $1: 1$ to receive usual cardiac care of periodic ECGs during office visits (control group) or usual cardiac care plus remote monitoring using the AliveCor device (intervention group; - Fig. 2). This device works with an accompanying smartphone application (app) to capture heart rate and rhythm via a single-lead ECG. Patients can use the app to document symptoms experienced during an ECG recording, or potential triggers of an AF episodes (e.g., exercise). iHEART intervention arm participants were asked to use the AliveCor device twice daily for 6 months but had the option of continuing beyond this period. This protocol was intended to facilitate early identification and treatment of AF, which can be difficult to capture with current standard of care measurements. ${ }^{14,35}$

A qualitative approach was used to allow for a richer, more nuanced understanding of the factors associated with sustained engagement. We recruited a convenience sample of iHEART intervention group participants who completed the trial within the past 2 months (to minimize recall bias). Providers and research coordinators were recruited because of their potential for insights into patient engagement stemming from their close connection to patients during the trial.

\section{Recruitment and Data Collection}

Institutional review board approval was obtained from Columbia University Medical Center. The primary author (M.R.) and the iHEART principal investigator (K.H.) identified potential participants and contacted them via telephone. Engaged patients, unengaged patients, and providers/research coordinators were recruited into separate sessions to facilitate candidness and comparison of engaged and unengaged patients. The level of engagement was determined by examining the Health Insurance Portability and Accountability Act-compliant, Web-based AliveCor portal. We defined the engaged patient as

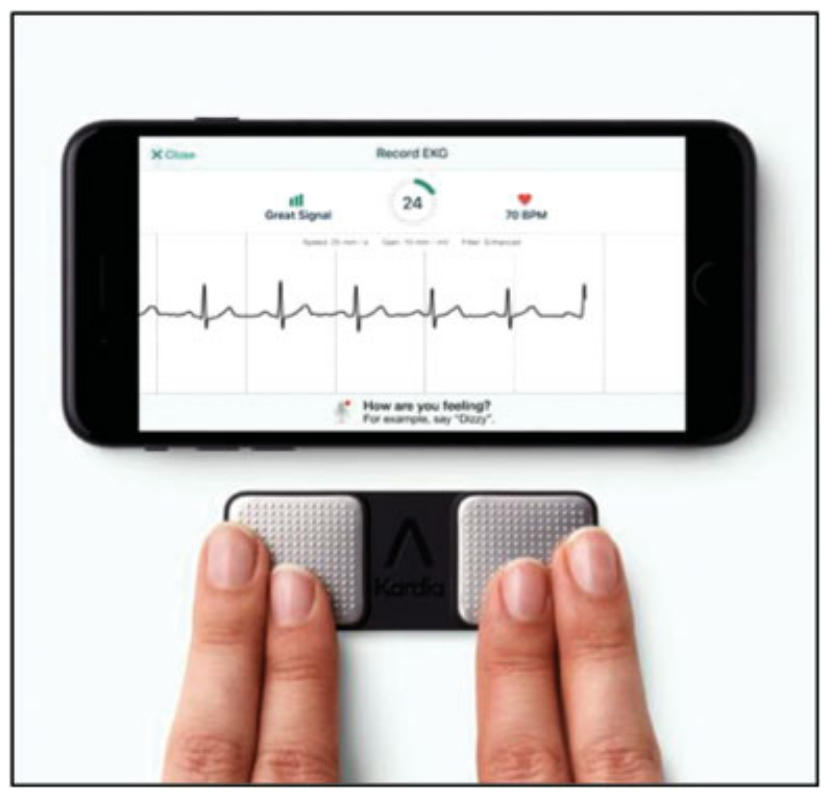

Fig. 2 AliveCor mobile electrocardiogram (ECG) monitor and smartphone application. one who used AliveCor at least once per day on average during the trial. We defined the unengaged patient as one who used the device less than once per day on average.

Informed consent was obtained at the beginning of each session. Semistructured focus groups and interviews were conducted and analyzed until theoretical saturation was reached. Discussions were guided by interview/focus group guides developed to elicit understanding on each factor in the adapted UTAUT model (e.g., perceived ease of use). Each session lasted 30 to 60 minutes and was conducted in a private space at a large, urban academic medical center or over the phone when needed due to travel or scheduling reasons. The primary author moderated all sessions. A second researcher (J.M.) was present for a subset of the sessions to ensure rigor in data collection. Neither researcher(M.R. or J.M.) was directly involved in the iHEART trial and did not know any subjects. Participants received a $\$ 20$ Visa gift card for participation. All focus groups and interviews were audio-recorded and transcribed verbatim. The primary author (M.R.) removed all personally identifiable information from all transcripts and checked them for accuracy prior to analysis.

\section{Data Analysis}

The transcripts were analyzed by directed content analysis. ${ }^{36}$ This method uses factors from a relevant theory to guide data collection and analysis. ${ }^{36,37}$ Following this approach, the primary author (M.R.) created a preliminary codebook of themes based on the factors described in the adapted UTAUT model, with separate sections for each participant group (engaged patients, unengaged patients, and providers/ research coordinators). The codebook was reviewed for content validity by J.M. All transcripts were then coded to this codebook by the primary author. New themes that emerged were reported separately. Two additional analysts (D.B. and M. B.), with no prior knowledge of the adapted UTAUT model, independently coded two transcripts using open coding (e.g., no a priori codes). This offered verification that the emergent themes they identified were congruent with the preliminary codebook, and that the codebook developed by the primary author was a valid coding instrument. The primary author then provided the preliminary codebook and they used directed coding to analyze three additional transcripts, while identifying and separating new themes that emerged.

At each stage, codes were compared. Discrepancies were discussed and resolved. Inter-rater reliability calculated to quantify coder agreement was high (0.87-0.98). In addition, all analysts identified and reported on similarities and differences between participant groups because both variability and consistency in perspectives were considered valuable in advancing understanding of the theoretical model. All data were analyzed using NVivo 11 (QSR International, Inc., Burlington, Massachusetts, United States).

\section{Results}

\section{Description of the Sample and Overall Engagement} We interviewed a total of 21 individuals: 13 patients ( 7 engaged, 6 unengaged); 6 providers; and 2 research coordinators. We 
conducted 13 individual interviews: 10 via phone with patients; 1 in-person with a patient; and 2 in-person with providers. We also conducted 2 in-person focus groups: 1 with 2 unengaged patients; and 1 with 4 providers and 2 research coordinators.

Providers in this study included 4 nurse practitioners and 2 physicians. They had, on average, 22.7 years (range: $20-27$ ) of clinical experience and 18.3 years (range: 13-25) working in the electrophysiology clinic from which iHEART participants were recruited. The 2 iHEART research coordinators reported 3 and 25 years of clinical research experience, respectively.

Patients were predominantly male (85\%) and middle- to older-age (mean: 65.3 years, range: 50-76 years), which reflects the demographics in the electrophysiology clinic from which they were recruited. Engaged and unengaged patients had approximately the same age and gender composition. Participants were asked a series of questions regarding their comfort with technology at baseline in the iHEART trial. All patients in this study reported owning a cell phone, with $78 \%$ owning a smartphone. All reported experience searching the Internet for health-related information, and all had a computer or tablet in their homes.

Engaged patients used AliveCor 31.2 times per month for an average of 11.9 months, compared with 24.1 times per month and for an average of 9.3 months among unengaged patients. Fig. 3 illustrates trajectories of AliveCor use over time, showing a clear difference in engagement between the two groups despite a high level of engagement overall.

\section{Factors Associated with Engagement in the UTAUT Model}

First, we describe themes associated with sustained engagement found in the adapted UTAUT model. We then describe emergent themes not specified in the adapted UTAUT model. Each theme and subtheme is presented in - Supplementary Table S1 (available in the online version) with illustrative quotes.

\section{Ease of Use}

Similarities in Ease of Use

Both engaged and unengaged patients reported that the AliveCor device was easy to use with minimal, if any, learning curve. They reported that data capture and sharing was simple with the device, and the lightweight design made it portable and therefore easy to capture ECGs virtually anywhere. Despite general ease of use, some technical challenges arose for most patients. The primary challenge reported was difficulty transmitting an ECG due to poor connectivity between fingertips and the device, or the device and the application. This led to poor-quality readings and vague output from the rhythm-identifying algorithm (e.g., "Unclassified"). Another problem described was background noise interference when symptoms were recorded through voice-enabled technology. Providers and research coordinators also reported that patients experienced these technical issues.

\section{Differences in Responses to Technical Issues}

The main difference between engaged and unengaged patients was attitude toward handling technical issues. All engaged patients reported on strategies they used for dealing with challenges related to transmission and connectivity, such as moving away from other electronic devices or cleaning their fingers. Some stated that this helped them avoid becoming anxious. Conversely, many unengaged

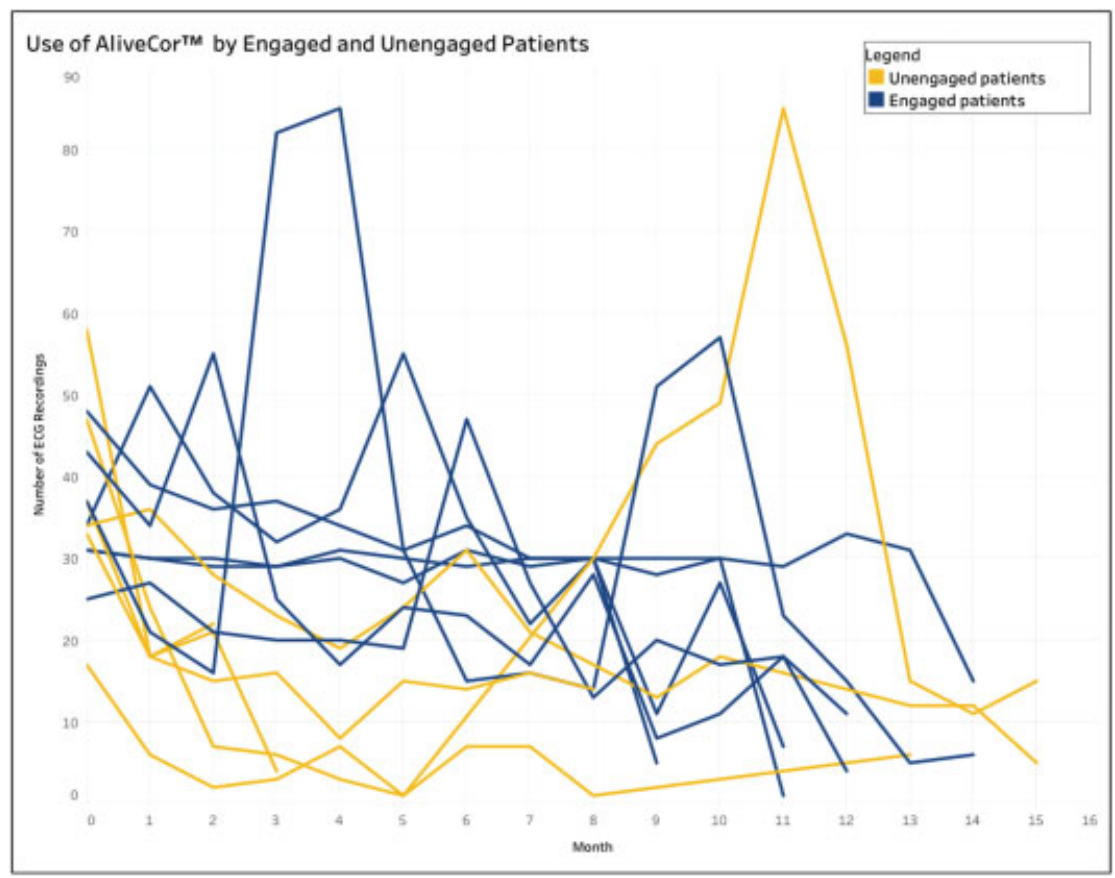

Fig. 3 Trajectories of engagement among iPhone Helping Evaluate Atrial Fibrillation Rhythm through Technology trial (iHEART) participants interviewed in this study $(n=13)$. 
patients expressed frustration and anxiety as a result of technical issues, for example: "I didn't feel safe in my ability to get accurate readings" - Patient 1 (unengaged).

\section{Differences in Health Care Provider Feedback}

Many engaged patients reported a small yet adequate amount of guidance from providers, which allowed them to handle abnormal readings and vague algorithm output: "I did have several false readings... [the doctor] said don't pay attention to those...He took that off the table for me to worry about" - Patient 9 (engaged). Most unengaged patients, however, reported little to no feedback from providers to help them overcome technical issues. For some, this was the direct reason for abandoning the device: "I stopped because it said unclassified and...nothing was happening. And I was going insane. What was going on? I wanted feedback" - Patient 11 (unengaged). All providers acknowledged this need but also pointed to time being a limiting factor in their ability to provide constant feedback to patients.

\section{Usefulness of the Technology}

Similarities in Usefulness of Identifying Rhythm

Most participants in both groups understood how difficult AF is to identify without an ECG. For this reason, they reported that AliveCor was useful in giving definitive rhythm identification, or "proof," as one patient called it. As a result, most patients stated that these data had a comforting effect, which providers corroborated.

\section{Differences in Insights and Perceived Value of the Data}

A major difference we found between engaged and unengaged patients was their ability to independently use the data they were collecting. Many engaged patients reported seeking further insights beyond basic heart rhythm, and stated that the value of the data was a reason for sustained use: "Sometimes I'll forget to take the medication but I never forget [AliveCor]... Because I value the feedback that it gives me tremendously" - Patient 13 (engaged). Conversely, many unengaged patients described confusion and difficulty interpreting their data: "When I stopped, I think part of it was getting the message unclassified kind of made wonder what the utility of this thing was" - Patient 10 (unengaged). Even if confusion did not arise, some unengaged patients did not attach value to insights beyond rhythm identification: "I'm blissfully unaware... I don't know if there's any other data that would be meaningful to me" - Patient 2 (unengaged).

\section{Differences in Health Care Provider Feedback}

Many engaged patients reported sharing insights about their data (described as "the signals and symptoms" by one patient) with their providers to tailor their self-management and medical care. Most providers recognized this supported the usefulness of the device: "We can try to sort out why they're having this rhythm problem and identify any triggers" - Provider 5. Most unengaged patients reported a need for interpretation to make the data useful, but indicated a lack of immediate feedback. This led to anxiety and even distrust toward providers and researchers: "It seemed like a one-way street where you guys were just taking my information and I'm out there on my own" - Patient 1 (unengaged). All providers recognized this, and some reported discouraging anxious patients from frequent monitoring that they felt may only worsen anxiety: "I, in fact, encourage them to not check it as often - it just doesn't serve any purpose besides potentially causing more anxiety about it" - Provider 5.

\section{Facilitating Conditions}

\section{AF Severity: Long AF Histories but Varying Proactive Behaviors}

Many patients in both groups reported living with AF for a long time but differed in how they reacted. Most engaged patients proactively changed behaviors, including healthier diets, abstaining from known AF "triggers" (e.g., drinking alcohol), and self-monitoring using AliveCor more frequently depending on clinical acuity: "I tried to use it every morning right after the ablation...As my rhythm returned normal it became something I checked less" - Patient 9 (engaged). In contrast, many unengaged patients reported being easily discouraged by their AF recurrence, which they said caused them to self-monitor less and instead rely on office visits with providers for rhythm monitoring: "I'm no longer in AF, at least, each time that I've been checked... I go in about every six weeks, just to be checked" - Patient 1 (unengaged).

Some providers observed that patients may appropriately decrease use over time if their heart rhythms became stable, indicating less AF severity: "For the clinical part, treatment is achieved and the patients are doing well. They're not less engaged, they're appropriately using it" - Provider 1 . They also pointed out, however, that this was only the case for patients who were truly clinically stable. If patients did not consider their clinical acuity, they could inappropriately discontinue use.

AF Knowledge: Differences in Uncovering Self-Knowledge Most patients had high levels of knowledge about AF in general. In fact, providers described the participating patients as "very sophisticated and educated" (Provider 6). However, patients' knowledge of personal physiology and self-management needs (self-knowledge) varied. Approximately half of engaged patients stated that their self-knowledge improved through self-monitoring: "I think that what changed was my sense of how this problem was affecting my day to day life" Patient 13 (engaged). Most unengaged patients, however, relied on providers to understand their unique physiology and needs "[My doctor] had told me that relatively speaking [caffeine is] the least effective trigger for me. He said alcohol is the worst and it definitely is, there's no question" - Patient 7 (unengaged).

\section{AF Symptoms: Driving Use for Unengaged Patients}

The majority of the patients in both groups understood that poor correlation between AF symptoms and AF episodes ${ }^{16,38,39}$ was a reason to use AliveCor to identify their true cardiac 
rhythm. Many engaged patients appropriately considered their actual ECG data versus their symptoms in determining whether to continue using AliveCor. Conversely, for many unengaged patients, use was driven by symptoms. They interpreted lack of symptoms as a sign of wellness and a reason to stop using AliveCor. Alternatively, some unengaged patients experienced symptoms that they attributed to AF when they were in a normal rhythm, causing them to use AliveCor too frequently. One unengaged patient described how perceived symptoms caused anxiety: "I probably use it too much because every time I have chest pain, I just pull it out. And after a while, I just stop that...Because I can't be doing it all the time" - Patient 5 (unengaged). Providers noticed this tendency: "They are not always in A fib when they do document symptoms... what they perceive to be something is not always the case" - Research Coordinator 2. Most unengaged patients expressed more confusion about their symptoms, describing them as unclear, inconsistently related to $\mathrm{AF}$, and shifting over time.

\section{Moderators: Age, Gender, and Experience with Technology}

Some providers and patients stated that they thought that age would influence ease of use and usefulness. Yet, no patient described their own age as being an impediment to AliveCor use, and most providers expressed confidence in their patients' ability to use the device regardless of age: "I've been surprised by how easily patients even in their 60's, 70's and 80 's have adopted using this" - Provider 6. Similarly, both engaged and unengaged patients described comfort with technology, and many reported tracking other aspects of their health with wearable devices and mobile applications. Even patients who did not consider themselves "tech savvy" expressed comfort using AliveCor commenting on its simple design: "I picked it up very easily. It was simple. And I'm not very good-I can't even program a remote control" - Patient 5 (unengaged). Providers and research coordinators agreed that tech savvy was unimportant if "enthusiasm for their care is there" - Research Coordinator 1. Unlike these other moderating factors, no participant explicitly discussed gender in the context of engagement with technology.

\section{New Findings}

Internal Motivation to Manage Health

Most patients in both groups expressed concern about their health. All considered themselves a part of the collaborative disease management process: "I'd like to live a long healthy life and being 50 years old, it's time to make a change. I'm hoping... I can continue to have a quality of life as I grow older" - Patient 4 (engaged). However, concern tended to escalate to anxiety for many unengaged patients, which providers corroborated: "Once they see something unusual from the baseline...they panic...they call right away" - Provider 1 .

\section{Relationship with Health Care Provider}

Most engaged patients described positive working relationships with their providers. Some stated that they had a strong relationship prior to using AliveCor, but most stated that the device and the data it generated improved the collaborative relationship. One patient said: "I feel like I am... 99\% in tune with them, or they with me, because it just gives them such important information" - Patient 6 (engaged). Some engaged patients also stated that the device improved collaboration between members of their care team. However, for unengaged patients, AliveCor did not facilitate collaboration with providers. They more frequently described relationships that were more patriarchal, and needing to advocate for themselves: "I wish they would listen to me... They're not looking at the whole picture" - Patient 5 (unengaged).

\section{Creating Supportive Environments}

Both engaged and unengaged patients described routines and reminders to integrate self-monitoring into daily habits. Many kept the device in the same place as a physical cue, to make it part of their "daily ritual," as one patient called it. Others took the device with them to spot-check if they experienced symptoms.

However, all engaged participants reported they maintained these environments, even when busy or travelling: "If I've missed the night I know to do it early in the morning and then just do twice the next day. It's rare...If I'm traveling I'll take it with me" - Patient 8 (engaged). Moreover, most engaged patients, as well as providers, described supportive networks of friends and family as critical: "Remembering was difficult but my wife was very helpful in the evenings and in the mornings" - Patient 13 (engaged). Alternatively, most unengaged participants described busy schedules and travelling as interfering with use: “On weekends I didn't do it...from the beginning I wasn't doing it every day. I guess, I just forgot it. I don't take it to work" - Patient 11 (unengaged). Few discussed support from family members, friends, or providers.

\section{Discussion}

\section{Summary of Findings}

In this study, we found similarities and differences between engaged and unengaged patients who used the AliveCor mHealth ECG technology to self-monitor their AF, which were corroborated by their providers and research coordinators. Patients were similar in many respects (e.g., most perceived AliveCor as useful on a basic level), but distinguishing patterns emerged that were both distinct and nuanced. For example, unengaged patients were generally frustrated by technical issues, confused by their heart rhythm data, and lacked support to help mitigate these issues. Conversely, most engaged patients were uninhibited by technical issues, able to interpret their data on deeper levels, and described supportive environments that promoted engagement.

\section{Revisiting the Concept of Engagement}

We found evidence that engagement is more complex than use or nonuse of mHealth technology. For instance, those patients who appropriately discontinue self-monitoring when clinically stable may be less frequent users over time, but their independent interpretation of their self- 
monitoring data would deem them engaged nonetheless. A clear definition of engagement with self-monitoring remains lacking in the informatics community. Part of the reason is because usage data remains the most common approach for measuring engagement but fails to capture its complexity. ${ }^{40,41}$ Future work should seek to develop a standardized definition and measure of engagement with self-monitoring that still accounts for nuances such as those described above.

\section{Fit with the Adapted UTAUT Model}

We found that the adapted UTAUT model adequately describes predictors of sustained engagement in this population. We found differences in the hypothesized predictors of sustained engagement between engaged and unengaged patients. For our population, the hypothesized moderators appeared less influential than we anticipated. This could reflect the limited variability within the study sample, as participants were similar in age and experience with technology, and were predominantly male.

Our findings suggest that four additional factors may contribute to sustained engagement in this population. Three of the four appear to operate as facilitating conditions. First, internal motivation to manage health was either a motivating force (as they were for engaged patients), or a mitigating force when concern escalated into anxiety (for some unengaged patients). Second, supportive environments, when present, fostered sustained engagement, and when absent was a reason for nonuse among unengaged patients. Third, patients' relationships with their providers, which ranged from collaborative (engaged patients) to deferential (unengaged patients), influenced sustained engagement. The fourth factor, feedback from providers, was discussed in the context of both perceived ease of use and perceived usefulness, and may moderate these predictors. In the iHEART trial, study coordinators and providers did not offer technical support follow-up, but participants had the option of contacting them for assistance. However, in our study, many unengaged patients reported feeling disconnected from any technical support or resources, leading to frustration and subsequent discontinuation of use with the AliveCor device.

The original UTAUT model contained factors that were condensed or eliminated in the adapted UTAUT model upon which we based our study. ${ }^{33}$ Three of the four additional factors that emerged in this study align with those eliminated from the original UTAUT model: internal values and motivations, supportive environments, and "social influence" (broadly aligning with the patient-provider relationship). ${ }^{29}$ Recent work revisiting the model supports inclusion of user attributes (e.g., attitudes), environmental attributes, and organizational attributes (including social influences). ${ }^{42,43}$ However, that work does not specifically address sustained engagement.

Thus, we conclude that sustained engagement is a multifaceted concept. Our study uncovered that the phenomenon of sustained engagement with self-monitoring involves interaction among three agents: the patient, the provider, and the technology. This conceptualization helps to explain why research that addressed these agents in isolation has demonstrated little success improving sustained engagement. ${ }^{27,28}$
Further, our findings suggest that all three must be incorporated into the design and evaluation of self-monitoring technologies that aim to facilitate sustained engagement.

\section{Relationship to Prior Work}

To our knowledge, this is the first study to use qualitative, primary source data to comprehensively describe factors related to sustained engagement with mHealth in a specific patient population. Jiang et al first used an adapted UTAUT model to predict sustained engagement among lung transplant patients. ${ }^{33}$ We extend their work by demonstrating the utility of the adapted model in a different patient population and, by doing so, identified additional factors relevant to sustained engagement.

Recent quantitative research more broadly identified factors (e.g., perceived usefulness) associated with engagement. ${ }^{44,45}$ Our qualitative work uncovered nuances within each factor, such as how previously unidentified variations in the depth of insights obtained from the data influenced how engaged and unengaged patients perceived usefulness. Such nuances may be testable in future quantitative studies in this population, using a larger sample size.

Internal motivation, a central construct in self-determination theory is critical for sustained engagement. ${ }^{46}$ Although this corresponds with a new factor we identified, "Internal Motivation to Manage Health," some responded to self-monitoring with anxiety that dampened their engagement over time. Self-determination theory therefore may require similar quantitative inquiry to understand how variation in internal motivation among specific patient groups contributes to the phenomenon of sustained engagement.

\section{Implications for Design}

Knowledge of the factors related to sustained engagement may be useful in tailoring self-monitoring applications. -Table 1 maps these factors to specific design implications. A first set of approaches focuses on feedback that unengaged patients reportedly lacked. These include links to online communities that might facilitate patient-to-patient communication, or application-based messaging with providers that might improve patient-provider communication and overall relationship. This is a controversial option, however, given the well-documented time, liability, reimbursement, and scope of practice issues that providers cite in response to application-based messaging. ${ }^{47}$

A second set of approaches focuses on automation to satisfy needs described by patients. These include tested solutions that have yet to be implemented for self-monitoring. For instance, clinical decision support, previously developed to support providers, ${ }^{48,49}$ could guide patients' interpretation and evaluation of their own clinical presentation through the data. Infobuttons merit application to mHealth applications. ${ }^{50,51}$ Interactive visualizations that help individuals make sense of large amounts of complex data have potential applications to PGHD. ${ }^{5,52}$ In this study, all subjects, including providers and research coordinators, noted that the feature for recording symptoms and triggers within AliveCor was difficult to use. If application design eases capture of AF symptoms and 
Table 1 Design implications from adapted UTAUT model

\begin{tabular}{|c|c|c|c|c|c|c|}
\hline & Feedback & & Automati & & & \\
\hline Factor & $\begin{array}{l}\text { Online } \\
\text { communities }\end{array}$ & $\begin{array}{l}\text { Messaging } \\
\text { with provider }\end{array}$ & $\begin{array}{l}\text { Patient } \\
\text { decision- } \\
\text { support }\end{array}$ & Infobuttons & $\begin{array}{l}\text { Additional } \\
\text { relevant } \\
\text { data } \\
\text { capture }\end{array}$ & $\begin{array}{l}\text { Interactive } \\
\text { data } \\
\text { visualizations }\end{array}$ \\
\hline Perceived ease of use & & & レ & レ & & \\
\hline Perceived usefulness & & & $\boldsymbol{\nu}$ & $\boldsymbol{\nu}$ & $\boldsymbol{\nu}$ & $\boldsymbol{\nu}$ \\
\hline AF severity ${ }^{\mathrm{a}}$ & & & 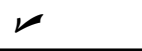 & & & レ \\
\hline AF knowledge ${ }^{a}$ & & & & レ & $r$ & $\nu$ \\
\hline AF symptoms ${ }^{a}$ & & & V & & $\boldsymbol{V}$ & レ \\
\hline Internal motivation ${ }^{a}$ & & & V & レ & & \\
\hline Relationship with provider ${ }^{a}$ & & レ & & & & \\
\hline Supportive environment $\mathrm{t}^{\mathrm{a}}$ & $r$ & レ & レ & $\swarrow$ & & \\
\hline Feedback and guidance ${ }^{\mathrm{b}}$ & $\boldsymbol{r}$ & $\boldsymbol{V}$ & & & & \\
\hline
\end{tabular}

Abbreviations: AF, atrial fibrillation; UTAUT, unified theory of acceptance and use of technology.

${ }^{a}$ Facilitating condition.

${ }^{\mathrm{b}}$ Moderator.

triggers, those data points could be triangulated with ECG data to discover individual manifestations of AF. Visualizations to enhance understanding of these triangulated data could improve AF management. ${ }^{52}$

\section{Implications for Research}

Our findings suggest several new lines of inquiry regarding sustained engagement. Providers observed there is a time to appropriately stop self-monitoring (if clinically stable for an extended period of time). For what length of time do patients actually need to self-monitor to receive a clinical benefit for specific conditions? Previous work has identified exact durations of remote monitoring necessary to diagnose or manage arrhythmias with implantable cardiac devices, ${ }^{53-55}$ but this issue remains inadequately studied in the self-monitoring space. This question should be considered in light of patients' perceptions of the need to continue self-monitoring, often based on symptoms, which may differ from their true clinical acuity. Evidence shows that AF episodes frequently do not correlate with perceived symptoms. ${ }^{16,38,39}$ Patients may be asymptomatic or experience vague symptoms that mimic those of comorbid conditions, and the type and severity of systems may change over time, which would necessitate continued use.

While we have identified several application design features that can target engagement, there remains the larger philosophical question of whether sustained engagement should be the goal for each patient. Patients and providers alike noted that anxiety can overcome utility for some patients. Others have found similar negative emotional responses to self-monitoring. ${ }^{56,57}$ While thoughtful design of applications that improve communication and information regarding the data may help, it will not mitigate anxiety for all patients. In such cases, the risk of continued anxiety, which itself is a risk factor for AF recurrence, may outweigh any clinical benefit of self-monitoring for the patient.

\section{Limitations}

This study had some limitations. First, while we attempted to classify patients' engagement from their behavior recorded in the AliveCor portal, more precise classification of engagement was not possible because raw usage data was not available. We also measured engagement over 1 year instead of 6 months (iHEART protocol) due to the high level of overall sustained engagement in the sample. As such, we may have inadvertently misclassified some patients' engagement. - Fig. 3 , which is a visualization of the raw usage data, shows clear differences between those participants that we initially identified as engaged and unengaged, suggesting that our classifications were accurate. Differences between engaged and unengaged and symptomatic and asymptomatic AF patients may warrant future confirmation using quantitative methods.

Second, this patient population was uniquely well-educated regarding their arrhythmia and highly engaged in their care overall. They were also predominantly male, middle- to older-age, and moderately to extremely comfortable with technology. Our sample therefore had little variability and tended toward high engagement with self-monitoring. While we made every attempt during our analysis to bracket biases that resulted from these sample characteristics, our findings are likely not generalizable to other patient populations, who may experience different barriers to sustained engagement. Therefore, this study underscores that theoretical models guiding data analysis always need to consider the unique patient population being studied.

\section{Conclusion}

This study provides insights on factors related to sustained engagement in a unique population of adults living with AF. We found evidence that the UTAUT model can serve as a valid framework for understanding sustained engagement, though it requires modifications to account for the patient 
population in consideration. The theory-driven findings we elicited can guide design and development of personalized mobile application interfaces for self-monitoring to engage adults living with AF for a sustained period of time. The UTAUT model also may guide establishment of parameters for sustained engagement for different patient populations. Theory-based evidence for application design is one approach for realizing the potential health benefits of PGHD collected with the mHealth technology.

\section{Clinical Relevance Statement}

PGHD is changing the paradigm of care for individuals living with chronic conditions for whom self-monitoring has the potential to improve clinical outcomes. This study validates a framework of sustained engagement that can be used to guide design and development of application interfaces that engage patients in the self-monitoring process. Through patient engagement, the promise of PGHD may soon be realized.

\section{Multiple Choice Questions}

1. In directed content analysis, qualitative data collection and analysis is guided by what?

a. Relevant health care policy.

b. Evidence-based medical guidelines.

c. A relevant theory.

d. Existing informatics ontologies.

Correct Answer: The correct answer is option c (see section "Data Analysis"). Direct content analysis starts with a theory as guidance for initial codes. Findings that emerge outside of this theory are also considered valuable and may suggest modifications to the theory.

2. Previous research has identified exact durations of monitoring necessary to detect or treat arrhythmias with what devices?

a. Implantable cardiac devices.

b. mHealth-based ECG monitors.

c. Both a and b.

d. Neither a nor b.

Correct Answer: The correct answer is option a (see section "Implications for Research"). Previous clinical research has determined the diagnostic yield and treatment-related benefits of monitoring with various implantable cardiac devices over specific time periods (e.g., 2 weeks of monitoring yields most diagnostic value). However, such research has not been conducted with smartphone-based ECG monitors, like AliveCor.

Protection of Human and Animal Subjects

This study received approval from the Institutional Review Board at Columbia University Medical Center.

\section{Funding}

M.R. is supported by the National Institute of Nursing Research (NINR; F31NR017313) and Jonas Center for Nursing Excellence. D.B. and M.B. are supported by
NINR (T32NR007969). K.T.H. is supported by NINR (R01NR014853).

\section{Conflict of Interest}

None.

\section{Acknowledgment}

The authors gratefully acknowledge the patients, providers, and research coordinators in this study who shared their experiences.

\section{References}

1 Bhavnani SP, Narula J, Sengupta PP. Mobile technology and the digitization of healthcare. Eur Heart J 2016;37(18):1428-1438

2 HIMSS. Definitions of mHealth. 2012. Available at: https://www. himss.org/definitions-mhealth. Accessed August 12, 2018

3 Silva BM, Rodrigues JJ, de la Torre Díez I, López-Coronado M, Saleem K. Mobile-health: a review of current state in 2015. J Biomed Inform 2015;56:265-272

4 Lai AM, Hsueh PS, Choi YK, Austin RR. Present and future trends in consumer health informatics and patient-generated health data. Yearb Med Inform 2017;26(01):152-159

5 Woods SS, Evans NC, Frisbee KL. Integrating patient voices into health information for self-care and patient-clinician partnerships: Veterans Affairs design recommendations for patientgenerated data applications. J Am Med Inform Assoc 2016; 23(03):491-495

6 Lavallee DC, Chenok KE, Love RM, et al. Incorporating patientreported outcomes into health care to engage patients and enhance care. Health Aff (Millwood) 2016;35(04):575-582

7 Arsoniadis EG, Tambyraja R, Khairat S, et al. Characterizing patient-generated clinical data and associated implications for electronic health records. Stud Health Technol Inform 2015; 216:158-162

8 Howie L, Hirsch B, Locklear T, Abernethy AP. Assessing the value of patient-generated data to comparative effectiveness research. Health Aff (Millwood) 2014;33(07):1220-1228

9 Shapiro M, et al. Patient-Generated Health Data: White Paper. 2012. Office of Policy and Planning, Office of the National Coordinator for Health Information Technology, Research Triangle Park, NC

10 Sanger PC, Hartzler A, Lordon RJ, et al. A patient-centered system in a provider-centered world: challenges of incorporating postdischarge wound data into practice. J Am Med Inform Assoc 2016; 23(03):514-525

11 Antman EM, Loscalzo J. Precision medicine in cardiology. Nat Rev Cardiol 2016;13(10):591-602

12 Hull S. Patient-generated health data foundation for personalized collaborative care. Comput Inform Nurs 2015;33(05):177-180

13 CDC. Atrial Fibrillation Fact Sheet; 2015. Available at: http:// www.cdc.gov/dhdsp/data_statistics/fact_sheets/fs_atrial_fibrillation.htm. Accessed September 3, 2018

14 Kirchhof P, Benussi S, Kotecha D, et al. 2016 ESC guidelines for the management of atrial fibrillation developed in collaboration with EACTS. Rev Esp Cardiol (Engl Ed) 2017; 70(01):50

15 Verdino RJ. Untreated atrial fibrillation in the United States of America: understanding the barriers and treatment options. J Saudi Heart Assoc 2015;27(01):44-49

16 Simantirakis EN, Papakonstantinou PE, Chlouverakis GI, et al. Asymptomatic versus symptomatic episodes in patients with paroxysmal atrial fibrillation via long-term monitoring with implantable loop recorders. Int J Cardiol 2017;231:125-130

17 Go AS, Hylek EM, Phillips KA, et al. Prevalence of diagnosed atrial fibrillation in adults: national implications for rhythm management and stroke prevention: the AnTicoagulation and Risk 
Factors in Atrial Fibrillation (ATRIA) Study. JAMA 2001;285(18): 2370-2375

18 Huxley RR, Lopez FL, Folsom AR, et al. Absolute and attributable risks of atrial fibrillation in relation to optimal and borderline risk factors: the Atherosclerosis Risk in Communities (ARIC) study. Circulation 2011;123(14):1501-1508

19 Turakhia MP, Kaiser DW. Transforming the care of atrial fibrillation with mobile health. J Interv Card Electrophysiol 2016;47(01): 45-50

20 Olgun Kucuk H, Kucuk U, Yalcin M, Isilak Z. Time to use mobile health devices to diagnose paroxysmal atrial fibrillation. Int J Cardiol 2016;222:1061

21 ONC. Conceptualizing a Data Infrastructure for the Capture, Use, and Sharing of Patient-Generated Health Data in Care Delivery and Research through 2024: Draft White Paper for a PGHD Policy Framework; 2016

22 Glasgow RE, Christiansen SM, Kurz D, et al. Engagement in a diabetes self-management website: usage patterns and generalizability of program use. J Med Internet Res 2011;13(01):e9

23 Mattila E, Orsama AL, Ahtinen A, Hopsu L, Leino T, Korhonen I. Personal health technologies in employee health promotion: usage activity, usefulness, and health-related outcomes in a 1year randomized controlled trial. JMIR Mhealth Uhealth 2013;1 (02): 16

24 Ford JH II, Alagoz E, Dinauer S, Johnson KA, Pe-Romashko K, Gustafson DH. Successful organizational strategies to sustain use of A-CHESS: a mobile intervention for individuals with alcohol use disorders. J Med Internet Res 2015;17(08):e201

25 Lasorsa ID Antrassi, P, Ajčević M, et al. Personalized support for chronic conditions. A novel approach for enhancing self-management and improving lifestyle. Appl Clin Inform 2016;7(03): 633-645

26 Khaneghah PA, Miguel-Cruz A, Bentley P, Liu L, Stroulia E, Ferguson-Pell M. Users' attitudes towards personal health records: a cross-sectional pilot study. Appl Clin Inform 2016;7(02):573-586

27 Shimada SL, Allison JJ, Rosen AK, Feng H, Houston TK. Sustained use of patient portal features and improvements in diabetes physiological measures. J Med Internet Res 2016;18(07):e179

28 King AC, Hekler EB, Grieco LA, et al. Harnessing different motivational frames via mobile phones to promote daily physical activity and reduce sedentary behavior in aging adults. PLoS One 2013;8 (04):e62613

29 Venkatesh V, Morris MG, David GB, Davis FD. User acceptance of information technology: toward a unified view. Manage Inf Syst Q 2003;27(03):425-478

$30 \mathrm{Kim} \mathrm{S}$, Lee KH, Hwang H, Yoo S. Analysis of the factors influencing healthcare professionals' adoption of mobile electronic medical record (EMR) using the unified theory of acceptance and use of technology (UTAUT) in a tertiary hospital. BMC Med Inform Decis Mak 2016;16:12

31 Lin B-S, Wong AM, Tseng KC. Community-based ECG monitoring system for patients with cardiovascular diseases. J Med Syst 2016; 40(04):80

32 Ma Q Chan AH, Chen K. Personal and other factors affecting acceptance of smartphone technology by older Chinese adults. Appl Ergon 2016;54:62-71

33 Jiang Y, Sereika SM, Dabbs AD, Handler SM, Schlenk EA. Acceptance and use of mobile technology for health self-monitoring in lung transplant recipients during the first year post-transplantation. Appl Clin Inform 2016;7(02):430-445

34 Sandelowski M. Whatever happened to qualitative description? Res Nurs Health 2000;23(04):334-340

35 Hickey KT, Hauser NR, Valente LE, et al. A single-center randomized, controlled trial investigating the efficacy of a mHealth ECG technology intervention to improve the detection of atrial fibrillation: the iHEART study protocol. BMC Cardiovasc Disord 2016; 16:152
36 Hsieh HF, Shannon SE. Three approaches to qualitative content analysis. Qual Health Res 2005;15(09):1277-1288

37 Graneheim UH, Lundman B. Qualitative content analysis in nursing research: concepts, procedures and measures to achieve trustworthiness. Nurse Educ Today 2004;24(02):105-112

38 Barrett PM, Komatireddy R, Haaser S, et al. Comparison of 24-hour Holter monitoring with 14-day novel adhesive patch electrocardiographic monitoring. Am J Med 2014;127(01):95.e11-95.e17

39 Dekker LR, Pokushalov E, Sanders P, Lindborg KA, Maus B, Pürerfellner $\mathrm{H}$. Continuous cardiac monitoring around atrial fibrillation ablation: insights on clinical classifications and end points. Pacing Clin Electrophysiol 2016;39(08):805-813

40 Steitz B, Cronin RM, Davis SE, Yan E, Jackson GP. Long-term patterns of patient portal use for pediatric patients at an academic medical center. Appl Clin Inform 2017;8(03):779-793

41 Williamson RS, Cherven BO, Gilleland Marchak J, et al. Meaningful use of an electronic personal health record (ePHR) among pediatric cancer survivors. Appl Clin Inform 2017;8(01): 250-264

42 Dwivedi YK, Rana NP, Jeyaraj A, Clement M, Williams MD, et al. Reexamining the unified theory of acceptance and use of technology (UTAUT): towards a revised theoretical model. Inf Syst Front 2017:1-16

43 Venkatesh V, Thong JYL, Xu X. Unified theory of acceptance and use of technology: a synthesis and the road ahead. J Assoc Inf Syst 2016;17(05):328-376

44 Hermsen S, Moons J, Kerkhof P, Wiekens C, De Groot M. Determinants for sustained use of an activity tracker: observational study. JMIR Mhealth Uhealth 2017;5(10):e164

45 Sharpe EE, Karasouli E, Meyer C. Examining factors of engagement with digital interventions for weight management: rapid review. JMIR Res Protoc 2017;6(10):e205

46 Coa K, Patrick H. Baseline motivation type as a predictor of dropout in a healthy eating text messaging program. JMIR Mhealth Uhealth 2016;4(03):e114

47 Reading MJ, Merrill JA. Converging and diverging needs between patients and providers who are collecting and using patientgenerated health data: an integrative review. J Am Med Inform Assoc 2018;25(06):759-771

48 Beeler PE, Bates DW, Hug BL. Clinical decision support systems. Swiss Med Wkly 2014;144:w14073

49 O'Sullivan D, Fraccaro P, Carson E, Weller P. Decision time for clinical decision support systems. Clin Med (Lond) 2014;14(04): 338-341

50 Teixeira M, Cook DA, Heale BSE, Del Fiol G. Optimization of infobutton design and implementation: a systematic review. J Biomed Inform 2017;74:10-19

51 Long J, Hulse NC, Tao C. Infobutton usage in patient portal MyHealth. AMIA Jt Summits Transl Sci Proc 2015;2015:112-116

52 Gotz D, Borland D. Data-driven healthcare: challenges and opportunities for interactive visualization. IEEE Comput Graph Appl 2016;36(03):90-96

53 Tung CE, Su D, Turakhia MP, Lansberg MG. Diagnostic yield of extended cardiac patch monitoring in patients with stroke or TIA. Front Neurol 2015;5:266

54 Turakhia MP, Hoang DD, Zimetbaum P, et al. Diagnostic utility of a novel leadless arrhythmia monitoring device. Am J Cardiol 2013; 112(04):520-524

55 Cheung CC, Kerr CR, Krahn AD. Comparing 14-day adhesive patch with 24-h Holter monitoring. Future Cardiol 2014;10(03):319-322

56 Ancker JS, Witteman HO, Hafeez B, Provencher T, Van de Graaf M, Wei E. "You Get Reminded You're a Sick Person": personal data tracking and patients with multiple chronic conditions. J Med Internet Res 2015;17(08):e202

57 Purtzer MA, Hermansen-Kobulnicky CJ. Optimizing the benefits of self-monitoring among patients with cancer. Oncol Nurs Forum 2016;43(06):E218-E225 